\title{
Review of Universal Modulator using Cordic Structure
}

\author{
Nandu K H $\mathbf{1}^{1}$, Mr. Santhosh $\mathrm{S}^{2}$, Mr. Praveen $\mathbf{J}^{3}$, Mr. Raghavendra Rao A \\ MTech $2^{\text {nd }}$ year, VLSI design and Embedded systems, Dept of ECE, AIET Mijar, Moodbidri, Karnataka, India ${ }^{1}$ \\ Assistant professor, Dept of ECE, AIET Mijar, Moodbidri, Karnataka, India ${ }^{2}$
}

Sr. Associate Professor, Dept of ECE, AIET Mijar, Moodbidri, Karnataka, India ${ }^{3}$

Sr. Associate Professor, HOD Dept of ECE, AIET Mijar, Moodbidri, Karnataka, India ${ }^{4}$

\begin{abstract}
In digital communication, there is a need of different modulation technique (such as ASK FSK PSK) to convey a message signal. A Universal modulation is the solution to obtain different modulation techniques. For the implementation of Universal Modulator on FPGA, different architecture have been proposed. One of the technique way to realize the Universal Modulator is Look up table (LUT). Here the CORDIC architecture is used for efficient realization of Universal Modulator. The coordinate rotation digital computer (CORDIC) algorithm is widely used in various technological fields such as digital signal processing (DSP), biomedical signal processing, robotics, communication systems, image processing etc. For simple shift and add operation, CORDIC based systems are increased drastically. For the realization of Amplitude modulation, Frequency modulation and Phase modulation, this Universal Modulation is used. The generation of SIN and COS output values will are tested for a given input angle $(\theta)$ value. The universal modulator will be designed around the CORDIC algorithms which can generate all most all digital modulation schemes such as ASK, PSK, FSK.
\end{abstract}

Keywords: FPGA Universal Modulator CORDIC ASK FSK PSK.

\section{INTRODUCTION}

The coordinate rotation digital computer (CORDIC) or result in a final angular argument of algorithm is a well-known iterative technique to perform zero(VECTORING).The algorithm is very attractive for various basic arithmetic operations including the hardware implementation because it uses only shift and computation of trigonometric functions, vector magnitude add operations to perform vector rotation. The rotation of estimation, polar to rectangular transformation etc. It is the two dimensional vector shown in fig $1 \mathrm{p}_{0}=\left[\mathrm{x}_{0} \mathrm{y}_{0}\right]$ preferred due to its simple shift-add operations, low cost through an angle, to obtain a rotated vector $\mathrm{p}_{\mathrm{n}}=$ and less complexity. In telecommunication the carrier $\left[\mathrm{x}_{\mathrm{n}} \mathrm{y}_{\mathrm{n}}\right]$ could be performed by the matrix product $\mathrm{p}_{\mathrm{n}}=$ waveform, that is modulated with an input signal for the purpose of conveying information. It is possible to generate the carrier directly by using an algorithm called CORDIC. CORDIC algorithm avoids the use of function generator which generates a carrier or different wave forms like sine wave, triangular wave, square wave etc. By using CORDIC algorithm, the digital modulation techniques can also be implemented like ASK, FSK, PSK, and QPSK. A carrier generator is generated by using CORDIC algorithm for digital modulation techniques by avoiding hardware complexity at very less power.

CORDIC may not be the fastest technique to perform these operations, it is attractive due to the simplicity of its hardware implementation, since the same iterative algorithm could be used for all these applications using the basic shift-add operations. Some of the typical approaches for reduced-complexity implementation are focused on minimization of the complexity of scaling operation the basic principle underlying the CORDIC-based computation, and present its iterative algorithm for different operating modes and planar coordinate systems.

The basic computing technique used in both the ROTATION and VECTORING modes in CORDIC is a step by step sequence of pseudo rotations which result in an overall rotation through the given angle (ROTATION)
$\mathrm{Rp}_{0}$,

where $\mathrm{R}$ is rotation matrix:

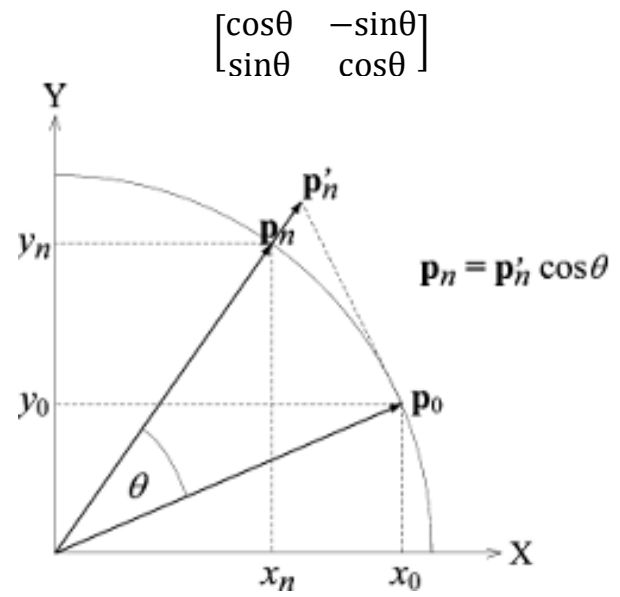

Fig 1:Two-dimentional Rotation vector

To achieve simplicity of hardware realization of the rotation, the key ideas used in CORDIC arithmetic are to (i) decompose the rotations into a sequence of elementary rotations through predefined angles that could be implemented with minimum hardware cost; and (ii) to avoid scaling, that might involve arithmetic operation, such as square-root and division. 


\section{II.}

\section{REVIEW WORK}

Mr. J.E. Volder in [1] has proposed the CORDIC Trigonometric Computing Technique the COordinate Rotation DIgital Computer (CORDIC) is a special purpose digital computer for real time computation. In this computer a unique computing technique is employed which is especially suitable for solving the trigonometric relationship. This involved phase coordinate rotation and conversion from rectangular to polar coordinates. In this paper only the trigonometric algorithm used in this computer and instrumentation of this algorithm.

The CORDIC computing technique is especially suitable for use in a special-purpose computer where the majority of the computation involves trigonometric relationship. In general, the ROTATION and VECTORING operations should be considered constant-length routine in which the number of word times per operation is equal to the word length. While not covered in this paper, similar algorithms have been developed for multiplication, division, conversion between binary and mixed radix systems, and extraction of square root, hyperbolic coordinate transformation exponentiation and generation of logarithm. It is believed that similar algorithm based on this fundamental concept of computation could be developed for many other computing requirements.

J. Walther in [2] has proposed a unified algorithm for elementary functions. In this paper they showed that, by varying a few simple parameters it could be used as a single algorithm for unified implementation of a wide range of elementary transcendental function. This function involving logarithm exponential and square roots along with those suggested by [1] Volder. Next, in the year 2009 marks the completion of 50 years of the invention of CORDIC by Jack E. Volder. The CORDIC lies in the fact that by simple shift-add operations. It can perform several computing tasks such as the calculation of trigonometric, hyperbolic and logarithmic functions, real and complex multiplications, division, square-root, solution of linear systems, eigenvalue estimation and many others.

Meher, P.K. et.al in [3]. Has designed a 50 Years of CORDIC: Algorithms, Architectures, and Applications. Year 2009 marks the completion of 50 years of the invention of CORDIC (COordinate Rotation DIgital Computer) by Jack E. Volder. The beauty of CORDIC lies in the fact that by simple shift-add operations, it can perform several computing tasks such as the calculation of trigonometric, hyperbolic and logarithmic functions, real and complex multiplications, division, square-root, solution of linear systems, eigenvalue estimation, singular value decomposition, QR factorization and many others. As a consequence, CORDIC has been utilized for applications in diverse areas such as signal and image processing, communication systems, robotics and 3-D graphics apart from general scientific and technical computation. In this article, they gave a brief overview of the key developments in the CORDIC algorithms and architectures along with their potential and upcoming applications.

In the last fifty years, several algorithms and architectures have been developed to speed up the CORDIC by reducing its iteration counts and through its pipelined implementation. Moreover, its applications in several diverse areas including signal processing, image processing, communication, robotics and graphics apart from general scientific and technical computations have been explored. Latency of computation, however, continues to be the major drawback of the CORDIC algorithm, since it does not have efficient algorithms for its parallel implementation. But, CORDIC on the other hand is inherently suitable for pipelined designs, due to its iterative behaviour, and small cycle time compared with the conventional arithmetic. For high-throughput applications, efficient pipelined-architectures with multiple-CORDIC units could be developed to take the advantage of pipeline ability of CORDIC, because the digital hardware is getting cheaper along with the progressive device-scaling. Research on fast implementation of shift-accumulation operation, exploration of new number systems for CORDIC, optimization of CORDIC for constant rotation have scope for further reduction of its latency. Another way to use CORDIC efficiently, is to transform the computational algorithm into independent segments, and to implement the individual segments by different CORDIC processors. With enhancement of its throughput and reduction of latency, it is expected that CORDIC would be useful for many high-speed and real-time applications. The areadelay-accuracy trade-off for different advanced algorithms may be investigated in detail and compared with in future work.

Peter Nilsson in [7] has proposed the complexity reduction in unrolled CORDIC architectures. Here he shows a novel methodology to reduce the complexity in unrolled CORDIC architectures. The methodology is based on eliminating the CORDIC stages starting from the first stage. As an example, a six stage CORDIC is used but the methodology is applicable on CORDICs with an arbitrary number of stages. The paper shows that the complexity can be reduced by $25 \%$.

Naresh $\mathrm{V}$ et.al in [8]. Has designed an area efficient multiplexer based CORDIC. In this, the efficacy of this approach is studied for the implementation on FPGA. For this study, both non pipelined and 2 level pipelined CORDIC with 8 stages and using two schemes - one using adders in all the stages and another using multiplexers in the second and third stages. It is found that the nonpipelined and pipelined CORDICs using multiplexer requires 1.6, 1.4 times lower area in Xilinx FPGA and 1.8, 1.6 times lower area than that using only adders. This is achieved without reduction in speed.

\section{PROPOSED WORK}

By observing all the authors works, proposes a Universal Modulator. Using the two levels pipelined CORDIC architecture which operates in Rotation mode works as a Universal Modulator.

The various input of the system is used for Amplitude modulation, Frequency modulation and Phase modulation. In this work a pipelined CORDIC of 2 stages of multiplex is proposed for efficient realization of Universal 
Modulator. Its performance is compared with the other CORDIC architecture like, Unrolled CORDIC and MUX based Unpipelined CORDIC.

\section{CONCLUSION}

All the authors have proposed different type of CORDIC architecture to solve the trigonometric relationship for realization of Universal Modulator. But that proposed technique having some trade off between speed and the delay. The aim of pipeline approach for MUX based CORDIC is to obtain sine and cosine of angle with an improved in area and delay.

\section{REFERENCE}

[1] J.E. Volder, "The CORDIC Trigonometric Computing Technique", IRE Transactions on Electronic computer, vol. EC- 8, pp. 330334,1959

[2] J. Walther, "a unified algorithm for elementary functions," proc. Spring joint comp. con \& vol.38, pp.379-385, 1971.

[3] Meher, P.K.; Valls, J.; Tso-Bing Juang; Sridharan, K.; Maharatna, K., "50 Years of CORDIC: Algorithms, Architectures, and Applications," Circuits and Systems I:Regular Papers, IEEE Transactions on , vol.56, no.9, pp.1893,1907, Sept. 2009I.S.Jacobs and C.P. Bean,

[4] "Fine particles, thin films and exchange anisotropy," in Magnetism, vol. III, G.T. Rado and H. Suhl, Eds. New York: Academic, 1963, pp. 271-350.

[5] Xueming Wei; Shengyuan Zhou, "A Novel Circuit Design Based CORDIC for OAM Modulator," Communications, Circuits and Systems, 2007. ICCCAS 2007. International Conference on, vol., no., pp.1197,1199, 11-13 July 2007.

[6] Vankka, J.; Kosunen, M.; Hubach, J.; Halonen, K., "A CORDICbased multicarrier QAM modulator," Global Telecommunications Conference, 1999. GLOBECOM'99 vol.1A, no., pp. 173,177 vol.1a, 1999

[7] Kangshun Li; Xiaoqiao Lu; Wensheng Zhang; Feng Wang, "Design and Implement of Digital Modulator Based on Improved DDS Technology and DSP Builder," Wireless Communications, Networking and Mobile Computing, 2009. WiCom '09. 5th International Conference on , vol., no., pp.1,5, 24-26 Sept. 2009.

[8] Peter Nilsson, "complexity reduction in unrolled CORDIC architectures" Electronics, circuits, and systems,2009.ICECS 2009, pp.868-871. Naresh V.; Venkataramani, B.; Raja, R., "An area efficient multiplexer based CORDIC," Computer Communication and Informatics (ICCCI), 2013 International Conference on , vol., no., pp.1,5, 4-6 Jan. 2013. 